\title{
Clinicoparasitological Study of Acute Severe Malaria in Children
}

\author{
${ }^{1}$ Tusti Ganguly, ${ }^{2}$ Asok Kumar Datta, ${ }^{3}$ Syamali Mandal, ${ }^{4}$ Pradip Kumar Das \\ ${ }^{1,2,4}$ Bankura Sammilani Medical College, West Bengal University of Health Sciences, Kolkata \\ ${ }^{3}$ M.G.M. Medical Colleges, Madhepura University, Bihar
}

\begin{abstract}
Malaria is a major disease in India and other Asian Countries next to Africa. Depending on the endemicity the incidence of malaria and its attack in pediatric population varies. The micro epedemiology of malaria is important. Situation of emergence of drug resistance in malaria is also alarming. There are reports of cases from new areas hitherto free from malaria and the change in the trend of the complications. Awareness of the relative prevalence of different complications in a particular geographic area could greatly facilitate the approach towards early diagnosis and prompt treatment. The study is conducted in Bankura Sammilani Medical College, Bankura, West Bengal. Total number of confirmed cases of malaria $(n=112)$ was taken from June.2010 to May 2011. The diagnosis was based on Clinical, Malaria antigen test and parasitological study. Among them severe malaria cases were selected based on the WHO criteria 2000 for severe malaria. Study done in relation to incidence, clinical features, complications and mortality of severe cases and compared those with non severe malaria cases. The mean age is about 6 years, $6.3 \%$ of cases occur in infancy, severe malaria in infancy is a dreaded complication. Male had higher frequency. Majority of severe malaria cases are due to p.falciparum. Commonest complication is cerebral malaria.
\end{abstract}

Key words: Antigen, Complication, Falciparum, Malaria, Parasite

\section{Introduction}

Nearly 300 to 400 million clinical episodes of malaria occur world wide, out of which 1.5 to 2 million die every year. Malaria causes one death every 30 seconds. $90 \%$ of these cases are from tropical Africa. The rest $10 \%$ occur in nearly a hundred countries, $2 / 3$ rd of these are reported in just three countries, i.e., Brazil, India, and Sri Lanka. Of all the cases of malaria in SE Asia, India contributes $80 \%{ }^{1}$

The clinical presentations of severe and complicated malaria vary in different parts of the world. In parts of the world where the endemicity of P. falciparum is stable, severe malaria is mainly a disease of childhood. In sub-Saharan Africa, where the disease is highly endemic, children below 5 years of age bear the brunt of the disease. They suffer more from cerebral malaria and anaemia resulting in very high mortality. Severe malaria is less common in older children and adults, because of acquisition of partial immunity. On the other hand, in places of lower endemicity, viz., SE Asia, the age shifts upwards and children above 5 years, and adults, suffer from severe falciparum malaria. ${ }^{2}$ However, even in these wide geographical areas, there are areas with very high to very low or seasonal transmission. Hence, more recently malaria is being perceived as a local and focal disease. In India, in Sundargarh district of Orissa, there are areas with intense transmission round the year with no serious or complicated cases. A few kilometres away, where transmission is low and seasonal, young adults suffer more from cerebral malaria and acute renal failure. All these show that micro-epidemiology is of immense importance. The situation is further complicated by the recent emergence of resistance to chloroquine and sulphadoxine-pyrimethamine therapy. In India, the resurgence of malaria, after the initial success of NMEP ( National Malaria Eradication Programme), has posed a great public health problem due to high mortality, morbidity, and the economic burden arising out of it. In India, the major areas with high endemicity are North-Eastern States, parts of Orissa, Bihar, Jharkhand, and West Bengal. Of late there are reports of cases from new areas hitherto free from malaria; and the change in the trend of the complications. Awareness of the relative prevalence of different complications in a particular geographic area could greatly facilitate the approach towards early diagnosis and prompt treatment ${ }^{3}$.

Therefore, there is a need to find out the clinical and parasitological features of the disease in this part of West Bengal, which is a low-tranmission area with bordering forest and hilly areas with higher-transmission. The main goal of this clinico-parasitological study was to explore the epidemiology of severe and uncomplicated malaria in patients admitted in a tertiary care hospital in eastern India and to comprehensively document on morbidity and mortality associated with severe malaria

\section{I.I Aims and Objectives}

1. To find out the proportion of severe malaria among all malaria cases

2. To study the spectrum of clinical manifestations of severe malaria.

3. To study the parasitological indices in children presenting with severe malaria. 
$4 \quad$ To identify the factors if any, which might have association with the development of complications and / or mortality in malaria.

\section{II.I Study Area}

\section{Materials and methods}

The study was conducted in the Pediatrics Inpatient Department of Bankura Sammilani Medical College and Hospital (BSMC\&H), Bankura, West Bengal. It has an API of $<2$ with a slide positivity rate of 1.54 $\& 1.14$ for the years $2010 \& 2011$, respectively.

\section{II.II Study Population}

One hundred and twelve cases of confirmed malaria were studied prospectively in the Pediatrics Inpatient Department of BSMC\&H from June 2010 to May 2011.

Inclusion criteria included age below or equal to 8 years, presence of malaria slide and / or RDT positivity. Among them, total one hundred and twelve patients were detected as suffering from malaria of which fortyseven patients with features suggestive of severe malaria were selected.

Exclusion criteria were age $>8$ years, absence of malaria blood slide or RDT positivity, other systemic illnesses like meningitis, poisoning, metabolic abnormalities, or known hepatic and renal disease.

\section{II.III Methods}

In the present study, diagnosis of malaria was done on the basis of demonstration of malaria parasite on blood slide examination of thick and thin smears, and/or malaria antigen positivity with RDTs. Among them severe malaria cases were selected based on the WHO criteria 2000 for severe malaria. ${ }^{4,5}$

\section{II.IV Study Technique}

1. All patients below or equal to 8 years admitted with clinical features compatible with the WHO criteria for severe malaria were included in the study.

2. An informed consent was taken from the parents /legal guardian of the child to include him/her in the study

3. A detailed history taking was done from a reliable caregiver of the child (mother/any other person) with a pre-designed, pre -tested schedule

4. Careful clinical examination was done with special emphasis on the complications Relevant clinicopathological, parasitological and biochemical investigations were performed .

\section{II.IV.I Parasitological diagnosis}

Collection of blood: Blood was collected by finger prick method for capillary blood. Films were made immediately after collection of blood.

Time of blood collection : as soon as malaria was suspected clinically, before initiating antimalarials Making thick and thin blood films Staining malaria parasites

\section{II.IV.II Reporting blood films for malaria parasites II.IV.III Reporting of Parasite count}

The parasite number were reported with the following plus scheme,mentioning the predominant morphological type of parasite seen. Gametocytes alone were not counted.

\begin{tabular}{|l|l|}
\hline parasite numbers & Report \\
\hline $1-10 / 100$ high power fields & + \\
\hline $11-100 / 100$ high power fields & ++ \\
\hline $1-10 /$ high power field & +++ \\
\hline$>10 /$ high power field & ++++ \\
\hline
\end{tabular}

At least 100 high power fields were examined before reporting as No Parasites Found (NPF).

II.IV.IV Diagnosis of malaria using a Rapid diagnostic Test (RDT)

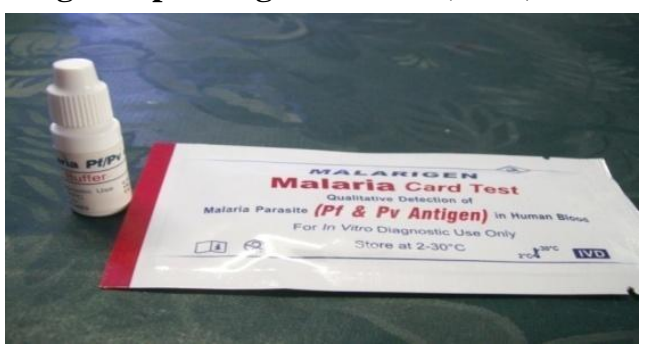

Picture1:Malarigen card RDT with buffer solution 

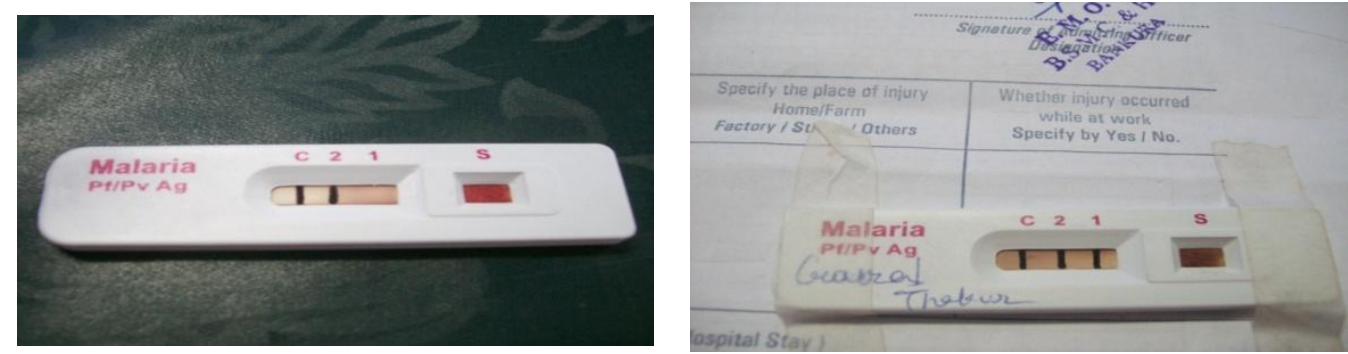

Picture 2.RDT positivity for P.vivax(left) and P.falciparum(right) by Malarigen card test

\section{II.IV.V Malarial morphology}

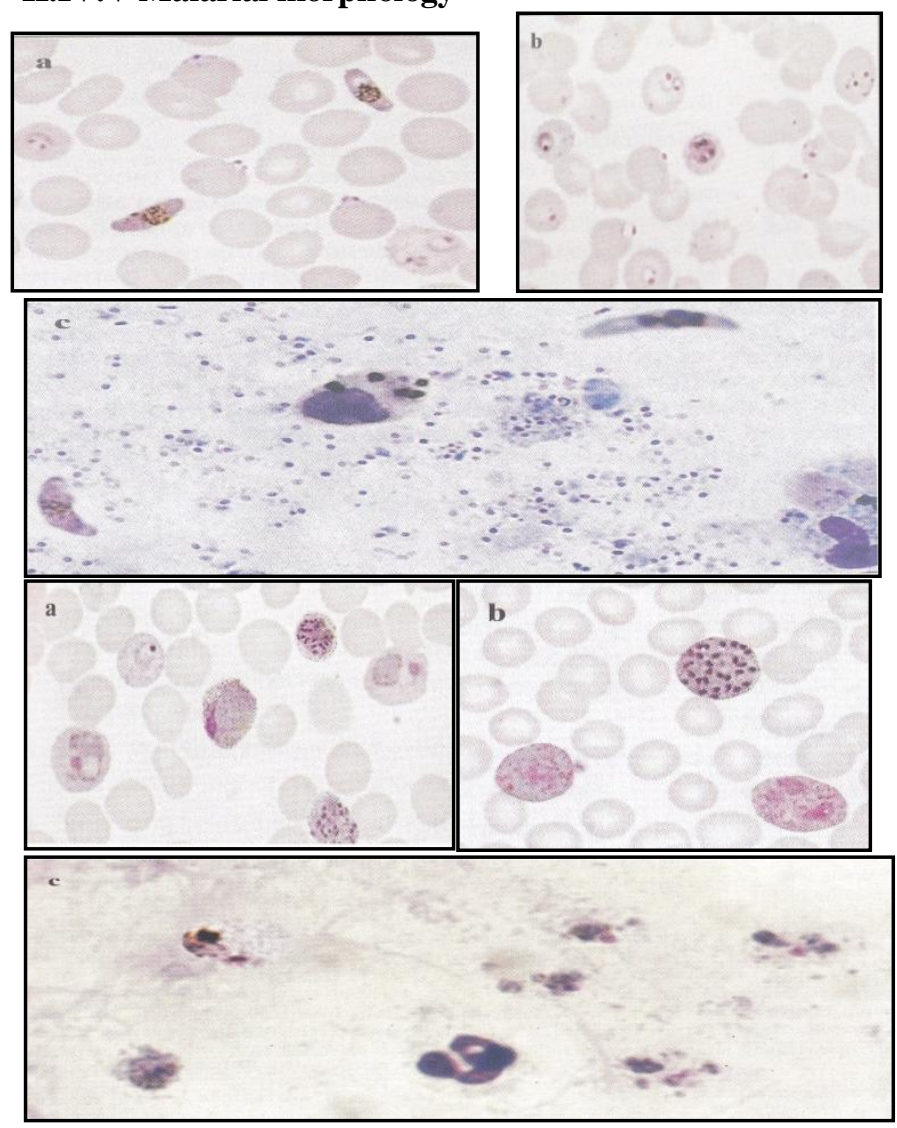

Figure I: P. falciparum

a. Trophozoites(ring forms) \& gametocytes on thin film

b. Young schizont (rare) \& trophozoites

c. Many trophozoites, gametocytes, and white cells containing pigment in thick film

Figure II: P. vivax

a. Trophozoites, gametocytes \&

schizonts on thin film

b. Schizont (mature), gametocyte \& trophozoites on thin film

c. Trophozoites in thick film

\section{Results and Analysis}

Distribution of cases according to age and sex: The median age of the children with severe malaria was 6 years. The age distribution of 65 cases of non severe malaria and 47 cases of severe malaria is given in Table 1. Ten deaths were recorded in the series, with an overall mortality rate of $21.3 \%$.

Table - Age distribution of cases

\begin{tabular}{|llllll|}
\hline Age $(\mathrm{yr})$ & $\begin{array}{l}\text { Cases of non } \\
\text { severe malaria }\end{array}$ & Severe malaria & Total & $\begin{array}{l}\text { Mortality in } \\
\text { severe patients }\end{array}$ & $\begin{array}{l}\text { P value for } \\
\text { mortality }\end{array}$ \\
$<1$ & $1(16.7)$ & $6(85.7 \%)$ & 7 & $1 / 6(16.7 \%)$ & 1.00 \\
$1-5$ & $21(52.5 \%)$ & $19(47.5 \%)$ & 40 & $3 / 19(15.8 \%)$ & 0.7180 \\
$5-8$ & $43(66.2 \%)$ & $22(33.8 \%)$ & 65 & $6 / 22(27.27 \%)$ & 0.4976 \\
TOTAL & 65 & 47 & 112 & $10 / 47(21.3 \%)$ & - \\
\hline
\end{tabular}

Table I shows Age Distribution of Severe and Non severe Cases of Malaria. Only severe cases $(n=47)$ were included in the statistical analysis to predict death. There was no mortality in the patients with non severe malaria. 
Malaria is more common among those between 5-8 years (46.8\%) as compared to 1-5 years (40.4\%). Few cases of malaria are found in infancy $(12.7 \%)$.*.Statistical analysis shows that malaria, when occurring in infancy, is more likely to be severe $(\mathrm{P}=0.0199)$. There was no significant difference in mortality rates among severe malaria cases in all age groups .

Table 2- Distribution of cases according to sex

\begin{tabular}{|llllll|}
\hline SEX & $\begin{array}{l}\text { Number of cases of } \\
\text { nonsevere malaria }(\% \\
\text { of total male/ female } \\
\text { cases) }\end{array}$ & $\begin{array}{l}\text { Number of cases of } \\
\text { severe malaria }(\% \text { of } \\
\text { total male/female } \\
\text { cases) }\end{array}$ & $\begin{array}{l}\text { Total number of in each sex } \\
\text { group }\end{array}$ & $\begin{array}{l}\text { Mortality } \\
\text { patients with } \\
\text { malaria (rate) }\end{array}$ \\
MALE & $38(58.5 \%)$ & $27(41.5 \%)$ & 65 & $6 / 27(22.2 \%)$ \\
FEMALE & $27(57.4 \%)$ & $20(42.6 \%)$ & 47 & $4 / 20(20 \%)$ \\
TOTAL & 65 & 47 & 112 & $10 / 47(21.3 \%)$ \\
\hline
\end{tabular}

The distribution of malaria cases among both sexes shows a slight male predominance in both severe (males$57.4 \%$ Vs females-42.6\%) and non severe malaria (males-58.5\% Vs females-41.5\%).

Table 3- Distribution of clinical features among malaria cases

\begin{tabular}{|lll|}
\hline Parameter & Cases & Percentage \\
Fever & 112 & $100 \%$ \\
Convulsion & 29 & $25.89 \%$ \\
Altered sensorium & 26 & $23.21 \%$ \\
Weakness & 15 & $13.39 \%$ \\
Palor & 41 & $36.6 \%$ \\
Splenomegaly & 12 & $10.71 \%$ \\
Hepatomegaly & 8 & $7.14 \%$ \\
Jaundice & 2 & $1.78 \%$ \\
Respiratory distress & 2 & $1.78 \%$ \\
Oligoanuria & 4 & $3.57 \%$ \\
Loose motion & 1 & $0.89 \%$ \\
Hyperpyrexia & 7 & $6.25 \%$ \\
Hemorrhage & 1 & $0.89 \%$ \\
\hline
\end{tabular}

Table 4- Patterns of laboratory abnormalities related to severe malaria

\begin{tabular}{|lll|}
\hline Parameter & Cases & $\%(\mathrm{n}=47)$ \\
Severe anemia $(\mathrm{Hb}<5 \mathrm{~g} / \mathrm{dl})$ & 12 & $25.5 \%$ \\
Thrombocytopenia & 1 & $2.1 \%$ \\
Hypoglycemia $(\mathrm{RBS}<40 \mathrm{mg} / \mathrm{dl})$ & 4 & $8.5 \%$ \\
Jaundice & 2 & $4.3 \%$ \\
$\uparrow$ Hepatic enzyme & 2 & $4.3 \%$ \\
Serum ceatinine $>2.5 \mathrm{mg} / \mathrm{dl}$ & 4 & $8.5 \%$ \\
Hyper paracytemia $>5 \%$ parasitized & 14 & $29.8 \%$ \\
RBC IN $P f ;>2 \%$ IN $P v)$ & & \\
\hline
\end{tabular}

Table 5- Parasitological parameters of non severe malaria

\begin{tabular}{|lll|}
\hline Causative agent & Cases & $\%$ of uncomplicated Cases \\
$P$. falciparum & 44 & $67.7 \%$ \\
P. vivax & 20 & $30.8 \%$ \\
P. falciparum + P.vivax & 3 & $4.6 \%$ \\
TOTAL & 65 & $100 \%$ \\
\hline
\end{tabular}

Table 6- Parasitological parameter of severe malaria

\begin{tabular}{|lll|}
\hline Causative agent & Cases & $\%$ of cases \\
$P$. falciparum & 40 & $85.1 \%$ \\
p.vivax & 4 & $8.5 \%$ \\
$P>$ falciparum $+P$. vivax & 3 & $6.4 \%$ \\
TOTAL & 47 & $100 \%$ \\
\hline
\end{tabular}


$P$. falciparum was the most common etiologic agent in both severe and non-severe malaria causing $75 \%$ of all malaria cases overall. However, P. falciparum infection was the predominant causative agent of severe malaria, causing $85 \%$ of cases. Only $8.5 \%$ of severe malaria cases were caused by $P$. vivax, while 6.4 $\%$ were due to dual infection by both species of parasites. *Therefore P. falciparum infection was more likely to cause severe malaria than P.vivax or mixed infection, and this was found to be statistically significant $(\mathrm{P}=0.0155)$

Table 7- Distribution of malaria parasite morphology among slide-positive cases

\begin{tabular}{|lll|}
\hline Morphological Features & No of Cases & $\begin{array}{l}\text { Percentage of total number of slide } \\
\text { positive cases (N=78) }\end{array}$ \\
Blood Slide Positive for P. falciparum & & $67.9 \%$ \\
Ring forms & 53 & $3.8 \%$ \\
Ring FormsS \& Gametocytes & 3 & \\
Blood Slide Positive for P.vivax & & $17.9 \%$ \\
Early/Late Trophozytes & 14 & $2.6 \%$ \\
Ring Forms \& Gametocytes & 2 & $1.3 \%$ \\
Ring Forms & 1 & \\
Blood Slide Positive for P. falciparum \& P. vivax & $3.8 \%$ \\
Ring Forms \& Trophozoites & 3 & $2.6 \%$ \\
Ring Forms & 2 & $100 \%$ \\
Total & 78 & \\
\hline
\end{tabular}

From the above table it is clear that the most common forms of the malaria parasite seen on BSE were ring forms of P. falciparum (67.9\%) and early and late trophozoites of P. vivax (17.9\%). Gametocytes of P. falciparum and P. vivax were seen accompanied by their asexual forms in 3.8 and 2.6 percent of slide positive cases, respectively. In cases of mixed infection with both species, ring forms of both types were found in all cases, with trophozoites of P.vivax seen in less than half $(40 \%)$ of such cases.

Table 8-Complications and mortality in severe malaria

\begin{tabular}{|lllll|}
\hline complications & $\begin{array}{l}\text { No(\%) } \\
(\mathrm{n}=47)\end{array}$ & of cases & $\begin{array}{l}\text { Mortality } \\
\text { rate })\end{array}$ & $(\%$ P value \\
Cerebral Malaria & $26(55.3 \%)$ & $9 / 26(34.6 \%)$ & 0.0115 \\
Convulsions $\geq 2$ & $29(61.7 \%)$ & $9 / 29(31.3 \%)$ & 0.0649 \\
Severe anemia & $12(25.5 \%)$ & $1 / 12(8.3 \%)$ & 0.4143 \\
Prostation & $15(31.9 \%)$ & $5 / 15(3.3 \%)$ & 0.2521 \\
Hyperparasitemia & $14(29.8 \%)$ & $9 / 14(64.3 \%)$ & $<0.00001$ \\
Hyperpyrexia & $7(14.9 \%)$ & $2(28.5 \%)$ & 0.6301 \\
Hypoglycemia & $4(8.5 \%)$ & $1(25 \%)$ & 1.00 \\
Acute renal failure & $4(8.5 \%)$ & $2(50 \%)$ & 0.1941 \\
Jaundice & $2(4.3 \%)$ & nil & \\
Pulmonary oedema & $1(2.1 \%)$ & $1(100 \%)$ & \\
Hemorrhage & 1 & nil & \\
& & & \\
\hline
\end{tabular}

\section{IV.I Demographic findings}

\section{DISCUSSION}

One hundred and twelve children below 8 years of age admitted to the Pediatrics Inpatient Department of Bankura Sammilani Medical College and Hospital during $1^{\text {st }}$ June 2010 to $31^{\text {st }}$ May, 2011 with malaria were studied prospectively for clinical, pathological and parasitological features of severe malaria, according to WHO criteria. The findings of these patients were carefully documented with utmost effort to eliminate errors. The results were tabulated and analysed statistically.

Of the 112 cases, 47 (41.9\%) patients conformed with the above mentioned criteria. 65 cases $(58.1 \%)$ did not have any of the criteria and were labelled as uncomplicated malaria. Of the total number of cases, 7 (6.3\%) cases of malaria were found in infants, $40(35.7 \%)$ cases among those between 1 year to below 5 years, and 65(57\%) cases were found in the age group of 5-8 years. The median age of malaria cases was 6 years. There were 65 males $(58.1 \%)$ and 47 females(41.9\%). There were 102 cases of malaria among nontribal classes and10 cases $(8.9 \%)$ among tribal classes . Ten deaths occurred, all in the severe malaria group, with an overall mortality rate of $21.3 \%$ in severe malaria. No neurologic of other sequelae were found at the time of discharge in any of the survivors of severe malaria. 
These demographic findings were in concordance with data from other studies in India as well as other regions of low endemicity for malaria. For example, in a population-based study done in Panna District of Madhya Pradesh ${ }^{6}$, analysis of age-specific data on malaria prevalence from study villages showed that the slide P. falciparum rate increased from $12.6 \%$ to $26.9 \%$ in children $<=1$ year of age (infants) to $35.6 \%$ in those $>1-4$ years of age to $39.4 \%$ in those $>4-8$ years of age, and then decreased to $31.3 \%$ in those $>14$ years of age $\left(\chi^{2}=\right.$ 32.2 , degrees of freedom $=4, P<0.0001$ ). In another study from Aligarh, Uttar Pradesh ${ }^{7}$, most cases were in 510 years age group $(56.5 \%)$ with $32.6 \%$ in the $1-5$ years age group. Males outnumbered females $(67.8 \%)$.

On the other hand, studies from hyperendemic and holoendemic regions for malaria showed a different picture. The mean age of children admitted with a primary diagnosis of malaria in an African study ${ }^{8}$ was 26 months, and 86 percent were under 4 years of age. There was no predominance of either sex at any age. In another study from Gabon ${ }^{9}$, the data for 7,980 children were analysed: $75 \%$ of these were less than 5 years of age. Among the total cases, $54.5 \%$ males and $45.5 \%$ females were found.

The number of severe malaria cases were stratified into age, sex and racial groups and compared with the numbers of uncomplicated malaria cases in the same groups. Age $<1$ year emerged as a statistically significant factor to be associated with severe malaria $(\mathrm{P}=0.0199)$, although it did not predispose to higher mortality $(\mathrm{P}=0.7481)$. No other age, sex or racial background was found to be significantly asoociated with severe malaria statistically.

The distribution of the cases with regards to their place of residence showed that $101(90.2 \%)$ cases were from the Bankura district, 10 cases $(8.93 \%)$ and 1 case $(0.89 \%)$ were from the neighbouring districts of Purulia and West Midnapore. Among the cases within Bankura district, 16 cases (14.3\%) were from Bankura, 11(9.8\%) from Chhatna, $9(8.1 \%)$ fron Onda, $7(6.3 \%)$ cases each from G.Ghati, Indpur which Taldangra are the P.S. areas nearest to Bankura, a total of $57(51.1 \%)$ of cases, rest of the cases were from different areas of Bankura district . Cases which were clustered around Bankura possibly because this hospital is the only State Government referral hospital in nearby areas.

\section{IV.II Clinical features}

In our study, the most common clinical features were fever(100\%), pallor(36.6\%), convulsions $(25.89 \%)$, altered sensorium $(23.21 \%)$ and weakness $(13.39 \%)$, splenomegaly $(10.71 \%)$ and hepatomegaly $>2 \mathrm{~cm}(7.14 \%)$. Severe malarial anemia (SMA) was found in $12(10.7 \%)$ of cases. The least common clinical features were loose motions and abnormal bleeding $(<1 \%$ each). Similar findings were reported from the study done by Peter, Manuel and Shetty, ${ }^{10}$ in Karnataka State of India in 2011.In this study, among all adults and children with P. falciparum malaria, the commonest symptom was fever (95\%) with chills and rigors. Among children, the next common symptoms were altered sensorium and irritability. $45 \%$ had cerebral malaria, among which half had seizures. Other common manifestations were reduced urine output $(35 \%)$ and vomiting (35\%). In another study from Jabalpur ${ }^{11}$, Madhya Pradesh the most common symptoms at presentation were fever $(80 \%)$, seizures (40\%), vomiting $(24 \%)$, headache $(16 \%)$, hepatosplenomegaly $(8 \%)$, and splenomegaly $(4 \%)$. Cerebral malaria was the commonest manifestation $(76.08 \%)$ of severe malaria in a study from Aligarh ${ }^{12}$. The presenting complaints in this study included fever in 46(100\%), unconsciouness in $39(84.8 \%)$, abnormal movements $(\mathrm{n}=16,34.8 \%)$, vomiting $(\mathrm{n}=8,17.3 \%)$, pallor $(n=7,15.2 \%)$, jaundice $(n=3,6.5 \%)$, cola colored urine $(n=3,6.5 \%)$, and headache, loose stools and irritability in 2 patients $(4.3 \%)$ each. Also in the study done in Cuttack, ${ }^{13}$ Orissa, the most common presentations were coma $(\mathrm{n}=159)$, prostration $(n=154)$, convulsions $(n=111)$, anemia $(n=107)$, and impaired consciousness $(n$ $=9$ ). This is in contrast to the studies in Africa ${ }^{14}$, which show severe anemia, to be the most frequent feature of severe malaria, followed by respiratory distress, cerebral malaria, hyperlactatemia and then hypoglycemia.

\section{IV.III Laboratory abnormalities}

Among the laboratory abnormalities in patients with severe malaria, the most common laboratory abnormalities were hyperparasitemia (29.8\%), severe anemia(25.5\%), hypoglycemia and raised serum creatinine (8.5\% each). Hyperbiliribinemia( $4.3 \%)$, elevated liver enzymes $(4.3 \%)$ and low platelet count $(2.1 \%)$ and were uncommon(table 6 and figure 7 of results section). These findings differed slightly from those in the study in Cuttack, ${ }^{13}$ where hyperparasitemia (31.8\%), severe anemia(28.6\%), hyperbilirubinemia(19.7\%), raised serum creatinine $(14.7 \%)$ and hypoglycemia $(6.4 \%)$ were the principal laboratoty derangements in severe malaria. In contrast, Marsh ${ }^{15}$ showed that severe malarial anemia (17.6\%), hypoglycemia (13.2\%), acidosis(63.6\%, $\mathrm{n}=110)^{*}$ and jaundice $(4.7 \%)$ were the common abnormalities.(*data from 110 out of 1866 patients included in the study was available).

Regarding parasitological test results, it was noticed that $P$. falciparum was the predominant causative agent of all malaria cases $(75 \%)$, while $85 \%$ of severe malaria cases were due to this species. The above data were similar to prevalence study of malaria in an area of low endemicity in India. ${ }^{16}$

Except for few reports of severe malaria caused by P.vivax from Bikaner ${ }^{17}$ and other places like Brazil ${ }^{18}$, Papua New Guinea ${ }^{19}$, severe malaria is known to be caused exclusively by $P$. falciparum . In this study, we found 
4(8.5\%) cases of severe malaria with P. vivax found on BSE( Blood Slide Examination) as well as P. vivax RDT( Rapid diagnostic test) positivity. Among them, 2 cases had multiple episodes of convulsions, of which one had altered sensorium for 90 minutes after convulsions with hyperpyrexia. There were 2 cases with severe anemia, and one case presented with severe weakness. There were also 3(6.38\%) patients with evidence of dual infection by both parasites; two of them had severe anemia, while one had hyperpyrexia with prostration. Although these numbers were very small and PCR was not possible to rule out falciparum malaria, yet these provide an indication of further studies into the clinical spectrum of vivax malaria.

Research into the sensitivity and specificity of diagnostic tests for malaria has revealed that BSE for malaria parasite is a more sensitive and specific test. We, however, found a large number of patients (30.4\%) who had the symptoms, signs and/or laboratory abnormalities of malaria without slide positivity for malaria parasite. Some of them had a history of intake of anti malarial drugs prior to hospital admission, while records were not available in many others. Therefore the high BSE negativity rate could be the reflection of empirical treatment of febrile illness with antimalarials, often in subtherapeutic doses, which led to treatment failure and/ or emergence of drug resistance. This may be the possible cause of clearance of parasites from the blood without complete abatement of symptoms.

Morphologically, ring forms $P$. falciparum (60.2\%) were the most commonly found forms of the malaria parasite on blood films. Next were early and late trophozoites of $P$. vivax (10.2\%).gametocytes of either species were found in $5.7 \%$ of cases.

\section{IV.IV Complications and mortality}

Complications and mortality patterns in severe malaria are varied, depending on transmission intensity (holo/hyper endemic Vs. meso/hypo endemic areas), age group studied (adults Vs children), and also in the same place over time. This is exemplified in the multicentric study done in Kenya, Gambia and Tanzania among populations with different transmission intensities, with $P$. falciparum prevalence rates in children between 2-10 years from $1 \%$ to $87 \%$. The observations summarized included overlapping communities seen at different times with very different estimates. One of the most striking observations was the relationship between increasing transmission intensity and the predominant age of pediatric malaria admissions. Among communities where PfPR2-10 ( Plasmodium falciparum prevalence rate between 2-10 years) is $\geq 40 \%$ more than $40 \%$ of malaria admissions to pediatric ward were infants, compared to only $10 \%$ in areas where PfPR2-10 is $<5 \%$. These observations are consistent with the view that the speed of acquired clinical immunity scales with the frequency of parasite exposure since birth. In this study series, cerebral malaria appeared to be a more common presentation among children hospitalized with

Malaria from lower intensity transmission settings compared to areas of high transmission; The proportion of children presenting with severe malaria anemia showed little variation across the range of transmission. Across this admission series of 10,642 and 10,742 cases of pediatric malaria respectively, cerebral malaria was reported among $4 \%$ and severe malaria anemia among $17 \%$ of admissions.

In another Multi centric study in Gabon ${ }^{20}$, Pediatric admission data were assembled from 13 hospitals serving 17 communities between 1990 and 2007. As transmission intensity declined a greater proportion of malaria admissions were in older children. There was a strong linear relationship between increasing transmission intensity and the proportion of pediatric malaria admissions in infancy $(\mathrm{R} 2=0.73, \mathrm{p}<0.001)$. Cerebral malaria was reported among $4 \%$ and severe malaria anemia among $17 \%$ of all malaria admissions. At higher transmission intensity cerebral malaria was a less common presentation compared to lower transmission sites. There was no obvious relationship between the proportions of children with severe malaria anemia and transmission intensity.

Another study was done in the highlands of Southwestern Uganda ${ }^{21}$, an area of extremely low malaria vector density, with estimated EIR <1/year with intermittent outbreaks, showed that severe malaria often occurred in persons older than 5 years of age. "Typical" signs like splenomegaly were frequently absent, prostration was the major manifestation, and other manifestations varied in frequency according to age.

In India, there are certain areas such as the Sundargarh district of Orissa ${ }^{22}$, where high malaria transmission intensity was recorded. A population based prevalence study done here demonstrated the presence of regions in the Indian subcontinent where P. falciparum is the primary cause of malaria and where malaria transmission rates are comparable to those found in many parts of Africa are present. Here the highest prevalence of parasitemia, as well as the frequency of clinical malaria episodes, was observed in children 1-5 years old. Both prevalence and incidence decreased with age. Malaria-related deaths and severe complications of malaria requiring hospitalization were not reported from the village during the study period. Other studies, like one from Rourkela, Orissa ${ }^{23}$ compared adult and child Plasmodium falciparum malaria patients hospitalized from 1995 to 1998, 608 had severe malaria and 83 died. Acute renal failure, jaundice and respiratory distress were common in adults whereas children frequently had severe anaemia. Cerebral malaria occurred equally in adults and children but recovery from coma was quicker in children. Multiple complications caused high mortality in adults. 
In contrast, a hospital study from Jabalpur ${ }^{24}$ showed that out of 564 severe malaria cases, 152 were having cerebral malaria of whom $39(25.6 \%)$ died. The majority of patients were in the age group of 16-40 years. In the study by $\mathrm{Huda}^{7}$ et al, Cerebral malaria was the most common complication (76.08\%), followed by severe anemia (34.78\%), hypoglycemia (21.73\%), jaundice(15.2\%), renal failure (8.69\%), black water fever(6.52\%) and algid malaria (4.3\%). In the study from Cuttack ${ }^{13}$, the complications in order of frequency were coma $(42.5 \%)$, prostration $(41.2 \%)$, hyperparasitemia $(31.8 \%)$, convulsions $(29.7 \%)$ severe anemia (28.8\%), impaired consciousness (25.4\%), jaundice (19.8\%), Acute renal failure (14.1\%), respiratory distress (7.2\%) and hypoglycemia (6.4\%). Changing trends of manifestation of severe malaria over time in the same location was reported in Bikaner, north-west India. ${ }^{25}$

In our study the commonest complications found were convulsions (61.7\%), coma(55.3\%), prostration(31.9\%), hyperparasitemia(29.8\%), severe anemia( 25.5\%), hyperpyrexia(14.8\%), hypoglycemia( $8.5 \%)$, ARF $(8.5 \%)$, respiratory distress $(4.2 \%)$, jaundice $(4.2 \%)$ and hemorrhage $(2.1 \%)$. These were similar to the findings of other studies from India.

In African children from a highly endemic area, impaired consciousness, jaundice, Respiratory distress (RD), and hypoglycemia are important predictors of death ${ }^{15}$. Renal failure is uncommon, and multiple organ failure has not been described. Indian studies ${ }^{13}$ have found four major predictors of mortality; RD, cerebral malaria, MODs, and hyper parasitemia. They also found a number of patients with 2 or more manifestations of severe malaria, and the risk of death increased with increasing number of co morbidities and/or involvement of $\geq 2$ organ systems. ${ }^{13}$

In the current study, we also found an overlap between patients with coma $(n=26)$, convulsions $(n=29)$, prostration $(n=15)$ and hyper parasitemia $(n=14) .5$ out of 47 patients had all of the four features, and all of them died. There were also 3 deaths out of 5 cases presenting with convulsions, coma and hyperparasitemia. Only 2 cases of prostration with coma and seizure were found with no deaths. There was only 1 death among 13 cases with coma and seizures. Thus, of the 22 patients who presented without coma and multiple seizures in severe malaria there were only 1 death out of total 10 deaths.

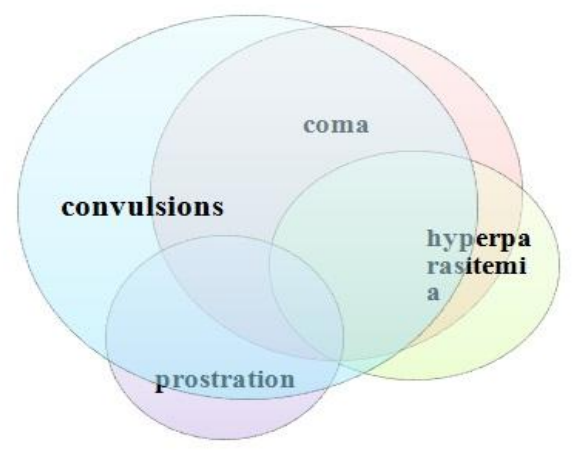

Figure 13. diagram showing overlapping relationships between parameters of severe malaria among study population.

\section{IV.V Cerebral malaria in the study group}

Cerebral malaria one of the most common and dangerous manifestations of complicated malaria. There are important differences between the patterns of disease in children compared with adults. Thus, defining the clinical spectrum of malaria in different age groups is necessary for studies conducted in areas of different malaria endemicity and transmission dynamics.

Cerebral malaria was the commonest form of severe malaria in the study group. The demographic data among the patients in our study did not reveal any special age, sex or racial predilection of cerebral malaria. All the patients were febrile, and $25(96.1 \%)$ had multiple $(\geq 2)$ episodes of seizures in the first 24 hours after admission. Other important associated clinic-parasitological features were hyperparasitemia (42.3\%), prostration (26.9\%), ARF (15.4\%), hypoglycemia (11.5\%), severe anemia (7.7\%) jaundice and hyperpyrexia (3.8\%) each. In a study from Madhya Pradesh ${ }^{28}$, the most common symptoms at presentation were fever $(80 \%)$, seizures $(40 \%)$, vomiting $(24 \%)$, headache $(16 \%)$, hepatosplenomegaly $(8 \%)$, and splenomegaly $(4 \%)$. Other severe complications included jaundice (26\%), ARF (22\%), respiratory distress (22\%), hypotension (17\%), hepatic encephalopathy (7\%), hypoglycemia (4\%), and hematuria (5\%). Also, $19 \%$ of patients had leukocytosis, $12 \%$ had leucopenia, and $27 \%$ had thrombocytopenia.

Overall there were 26 patients with cerebral malaria with 9 deaths, a mortality rate if $34.6 \%$. The case fatality rate was $21 \%$ in the study by $\mathrm{Jain}^{26}$ et al. However, the mortality caused by Cerebral Malaria varies from $8 \%$ to $33 \%$ in various countries: $8 \%$ in Zambia, ${ }^{27} 13.9 \%$ in Thailand, ${ }^{28} 32.7 \%$ in Bangladesh, ${ }^{29}$ and $23-33 \%$ in India. ${ }^{30}$ Mortality was higher among cerebral malaria patients in the presence of hyperparasitemia $(\mathrm{P}<0.00001)$, seizures $(\mathrm{P}=0.0118)$ and prostration $(\mathrm{P}=0.0028)$. Other findings were not associated with significantly increased 
mortality. This was similar to the study in Malawi, ${ }^{30}$ where hypoglycemia, hyperparasitemia, hyperleucocytosis, age $\leq 3$ years, deep coma and convulsions were poor prognostic markers. Risk factors strongly related to mortality in children presenting with neurologic involvement in Kenya ${ }^{8}$ included respiratory distress $(\mathrm{P}<0.001)$, hypoglycemia $(\mathrm{P}<0.001)$ and deep coma $(\mathrm{P}<0.008)$. Severe anemia $(\mathrm{Hb}<5 \mathrm{~g} / \mathrm{dl})$ and jaundice were not significantly related to mortality.

Furthermore, in this study, a large number of patients died soon after hospitalization. More than $40 \%$ of the cerebral malaria related deaths occurred within 24 hours of admission, before the patients had complete therapy. There is clearly a need for prompt institution of therapy for any subject with multiple malaria complications presenting at primary or secondary levels of the health care system. Cerebral malaria was the most common form of severe malaria $(55.3 \%)$ in this study, and it was seen to occur almost equally in all age, sex and racial groups.

All of the cases of cerebral malaria were caused by P.falciparum. Cerebral malaria was often associated with other complications, of which convulsions (96.1\%), hyperparasitemia (42.3\%) and prostration(26.9\%) were most common. ARF (15.4\%) and hypoglycemia (11.5\%) were not uncommon.

\section{Conclusion}

We have attempted to find out the spectrum of clinical and parasitological features in severe malaria in and around Bankura and to identify the factors which might be related with adverse outcome in malaria. Multiple clinical presentations were seen, the most common being fever (100\%), pallor (36.6\%), convulsions (25.89\%), altered sensorium $(23.21 \%)$ weakness $(13.39 \%)$ and hyperpyrexia $(6.25 \%)$. Hyperparasitaemia $(29.8 \%)$, severe anemia (25.5\%), hypoglycemia and raised creatinine $(8.5 \%)$ each were the commonest laboratory derangements.

The causative agent was $P$. falciparum in $75 \%$ and $85 \%$ of total and severe malaria cases respectively. $8.5 \%$ of severe malaria case were caused by $P$. vivax, and $6.4 \%$ due to mixed infection by both $P$. falciparum and P.vivax. There were many complications among the severe malaria cases; convulsions $(61.7 \%)$, coma (55.3\%), hyperparasitaemia $(31.9 \%)$, prostration $(29.8 \%)$ and severe anemia $(25.5 \%)$ were the commonest.

\section{Acknowledgement}

We acknowledge Professor Monoj Kumar Chowdhury, The Principal of Bankura Medical College for his constant inspiration and help during this study.

\section{References}

[1] WHO SE Asia. Implementation of collaborative activities on Roll back Malaria in SE Asia region. June, 2000 New Delhi 1 -21.

[2] World Health Organization Severe falciparum malaria. Trans R Soc Trop Med Hyg. 2000;94(suppl 1):S1-S90.[PubMed]

[3] A global strategy for malaria control. Switzerland : WHO 1993

[4] World Health Organization Severe and complicated malaria. Trans R Soc Trop Med Hyg. 1990;84(suppl 2):S1-S65.

[5] World Health Organization Severe falciparum malaria. Trans R Soc Trop Med Hyg. 2000;94(suppl 1):S1-S90.[PubMed]

[6] Neeru Singh, S.K. Chand, A.K. Mishra, Praveen K. Bharti, M. P. Singh, T. P. Ahluwalia. Epidemiology of Malaria in an Area of Low Transmission in Central India. Am. J. Trop. Med. Hyg., 75(5), 2006, pp. 812-816

[7] S.N. Huda, T. Shahab, S.M. Ali, K. Afzal and H.M. Khan . A comparative Clinical Trial of Artemether and Quinine in Children With Severe Malaria. Departments of Pediatrics and Microbiology, Jawaharlal Nehru Medical College, AMU, Aligarh, U.P., India. Indian Pediatrics 2003; 40:939-945

[8] Kevin Marsh, Dayo Forster, Catherine Waruiru, Isiah Mwangi, Maria Winstanley,Victoria Marsh, Charles Newton, Peter Winstanley, Peter Warne, Norbert Peshu, Geoffrey Pasvol, Robert Snow Indicators of Life-threatening Malaria in African Children. N Engl J Med 1995; 332:1399-1404 May 25, 1995

[9] Arnaud Dzeing-Ella, Pascal C Nze Obiang, Rose Tchoua, Timothy Planche, Béatrice Mboza, Monique Mbounja, Ulrich MullerRoemer, Joseph Jarvis, Eric Kendjo, Edouard Ngou-Milama, Peter G Kremsner, Sanjeev Krishna and Maryvonne Kombila. Severe falciparum malaria in Gabonese children: clinical and laboratory features. Malaria Journal 2005, 4:1 doi:10.1186/14752875-4-1

[10] George Peter, Alexander Lobo Manuel, Shetty Anil. Study comparing the clinical profile of complicated cases of Plasmodium falciparum malaria among adults and children. Asian Pacific Journal of Tropical Disease (2011)35-37

[11] Vidhan Jain, Avinash C. Nagpal, Pradeep K. Joel, Manmohan Shukla, Mrigendra P. Singh, Rasik B. Gupta, Aditya P. Dash, Saroj K. Mishra, Venkatachalam Udhayakumar, Jonathan K. Stiles, AND Neeru Singh Burden of Cerebral Malaria in Central India (2004-2007) Am. J. Trop. Med. Hyg., 79(4), 2008, pp. 636-642

[12] S.N. Huda, T. Shahab, S.M. Ali, K. Afzal and H.M. Khan

[13] A comparative Clinical Trial of Artemether and Quinine in Children With Severe Malaria

[14] Departments of Pediatrics and Microbiology, Jawaharlal Nehru Medical College, AMU, Aligarh, U.P., India.

[15] Indian Pediatrics 2003; 40:939-945

[16] Radha Tripathy, Sailajanandan Parida, Leena Das, Debi Prasad Mishra, Diptimayee Tripathy, Mangala Charan Das, Hegang Chen, James H. Maguire and Pinaki Panigrahi . Clinical Manifestations and Predictors of Severe Malaria in Indian Children. Pediatrics 2007

[17] Arnaud Dzeing-Ella, Pascal C Nze Obiang, Rose Tchoua, Timothy Planche, Béatrice Mboza, Monique Mbounja, Ulrich MullerRoemer, Joseph Jarvis, Eric Kendjo, Edouard Ngou-Milama, Peter G Kremsner, Sanjeev Krishna and Maryvonne Kombila Severe falciparum malaria in Gabonese children: clinical and laboratory features. Malaria Journal 2005, 4:1 doi:10.1186/14752875-4-1http://www.malariajournal.com/content/4/1/1 
[18] Kevin Marsh, Dayo Forster, Catherine Waruiru, Isiah Mwangi, Maria Winstanley,Victoria Marsh, Charles Newton, Peter Winstanley, Peter Warne, Norbert Peshu, Geoffrey Pasvol, Robert Snow Indicators of Life-threatening Malaria in African Children. N Engl J Med 1995; 332:1399-1404May 25, 1995

[19] Neeru Singh,S.K. Chand, A.K. Mishra, Praveen K. Bharti, M. P. Singh, T. P. Ahluwalia. Epidemiology of Malaria in an Area of Low Transmission in Central India. Am. J. Trop. Med. Hyg., 75(5), 2006, pp. 812-816

[20] Dhanpat K. Kochar, Ashish Das, Sanjay K. Kochar, Vishal Saxena,Parmendra Sirohi, Shilpi Garg, Abhishek Kochar, Mahesh P. Khatriand Vikas Gupta.

[21] Severe Plasmodium vivax Malaria: A Report on Serial Cases from Bikaner in Northwestern India. Am J Trop Med HygFebruary 2009 vol. 80 no. $2194-198$

[22] 18. Bruno B Andrade, Antonio Reis-Filho, Sebastião M Souza-Neto, Jorge Clarêncio, Luis MA Camargo, Aldina Barral and Manoel Barral-Netto

[23] Severe Plasmodium vivax malaria exhibits marked inflammatory imbalance. Malaria Journal 2010, 9:13 doi:10.1186/1475-28759-13

[24] Blaise Genton, Valérie D'Acremont, Lawrence Rare,Kay Baea, John C. Reeder, Michael P. Alpers, Ivo Müller

[25] Plasmodium vivax and Mixed Infections Are Associated with Severe Malaria in Children: A Prospective Cohort Study from Papua New Guinea

[26] http://www.plosmedicine.org/article/info:doi/10.1371/journal.pmed.0050127

[27] Emelda A Okiro, Abdullah Al-Taiar, Hugh Reyburn, Richard Idro,James A Berkley, and Robert W Snow. Age patterns of severe paediatric malaria and their relationship to Plasmodium falciparum transmission intensity

[28] Malaria Journal 2009, 8:4 doi:10.1186/1475-2875-8-4 article is available from: http://www.malariajournal.com/content/8/1/4

[29] Richard Idro, Edward Bitarakwate, Sam Tumwesigire, and Chandy c. John Clinical manifestations of severe malaria in the highlands of Southwestern Uganda.

[30] Am. J. Trop. Med. Hyg., 72(5), 2005, pp. 561-567

[31] 22. Surya K. Sharma, Rana Chattopadhyay, Kausik Chakrabarti, Sudhanshu S. Pati,Vinod K. Srivastava, Prajesh K. Tyagi, Sanjib Mahanty, Saroj K. Misra, Tridibes Adak,Bhabani S. Das, And Chetan E. Chitnis

[32] Epidemiology of Malaria Transmission and Development of Natural Immunity in a Malaria-Endemic Village, San Dulakudar, in Orissa State, India

[33] Am. J. Trop. Med. Hyg., 71(4), 2004, pp. 457-465

[34] Mohanty S, Mishra SK, Pati SS, Pattnaik J, Das BS. Complications and mortality patterns due to Plasmodium falciparum malaria in hospitalized adults and children, Rourkela, Orissa, India. 2003. Trans R Soc Trop Med Hyg97: 69-70

[35] Shukla MM, Singh N, Singh MP, Tejwani BM, Srivastava DK, Sharma VP.Source. Cerebral malaria in Jabalpur, India Indian J Malariol. 1995 Jun;32(2):70-5.. Malaria Research Centre, (Field Station), Medical College Building, Jabalpur, India.

[36] D.K. Kochar, S.K. Kochar, R.P. Agrawal, M. Sabir, K.C. Nayak, T.D. Agrawal, V.P. Purohit \& R.P. Gupta The changing spectrum of severe falciparum malaria: a clinical studyfrom Bikaner (northwest India ).J Vect Borne Dis 43, September 2006, pp. $104-108$

[37] 26.Vidhan Jain, Avinash C. Nagpal, Pradeep K. Joel, Manmohan Shukla, Mrigendra P. Singh, Rasik B. Gupta, Aditya P. Dash, Saroj K. Mishra, Venkatachalam Udhayakumar, Jonathan K. Stiles, AND Neeru Singh

[38] Burden of Cerebral Malaria in Central India (2004-2007)

[39] Am. J. Trop. Med. Hyg., 79(4), 2008, pp. 636-642

[40] Olweny CL, Chauhan SS, Simooya OO, Bulsara MK, Njelesani EK, Van Thuc H, Adult cerebral malaria in Zambia: preliminary report of clinical findings and treatment response.1986. J Trop Med Hyg89: 123-129

[41] Warrell DA, White NJ, Warrell MJ, 1982. Dexamethasone deleterious in cerebral malariaBMJ285: 1652

[42] Shukla MM, Singh N, Singh MP, Tejwani BM, Srivastava DK, Sharma VP.Source.Cerebral malaria in Jabalpur, India Indian J Malariol. 1995 Jun;32(2):70-5.. Malaria Research Centre, (Field Station), Medical College Building, Jabalpur, India

[43] Kochar DK, Sirohi P, Kochar SK, Budania MP, Lakhotia JP .Dynamics of malaria in Bikaner, Rajasthan, India (1975- 2006). 2007. J Vector Borne Dis.44: 281-284 